EXTENDED REPORT

\title{
Impact of musculoskeletal disorders on quality of life: an inception cohort study
}

\author{
C H Roux, F Guillemin, S Boini, F Longuetaud, N Arnault, S Hercberg, S Briançon
}

Ann Rheum Dis 2005;64:606-611. doi: 10.1136/ard.2004.020784

See end of article for authors' affiliations

.....................

Correspondence to: Professor F Guillemin,

Ecole de Santé Publique, Faculté de Médecine, 9 Avenue de la Forêt de Haye, F-54505

Vandoeuvre-les-Nancy, France; francis.guillemin@ medecine.uhp-nancy.fr

Accepted 12 August 2004 Published Online First 2 December 2004

\begin{abstract}
Objective: To assess longitudinally the impact of new onset musculoskeletal (MSK) disorders on quality of life (QoL).

Methods: An inception cohort of 1202 subjects in France aged 45-60 years was determined to be free of MSK problems at baseline. Over 28 months of follow up between 1996 and 1998, 310 were diagnosed with MSK disorders and matched for age and sex with 620 healthy controls. The impact of the MSK disorder onset on QoL was assessed by the change in SF-36 dimension scores over time, using a linear mixed ANOVA model to compare the groups.

Results: The incidence of MSK disorder was $13.6 \%$ per person-year in the spine, $4.2 \%$ per person-year in a joint, and $4.6 \%$ per person-year at an extra-articular site. The greatest change in QoL was a 10 point drop in the 100 point SF-36 bodily pain dimension scale in the MSK group. Compared with controls, subjects with an MSK disorder had significantly greater reductions in the following dimensions: bodily pain (a -7.4 point difference in change), vitality $(-2.7)$, general health $(-1.8)$, and physical functioning (-1.3). Within the MSK group, chronic disorders had a greater impact than acute ones on the physical functioning $(-2.1)$, role emotional $(-8.4)$, and social functioning $(-5.9)$ dimensions.

Conclusion: New onset MSK disorders have a marked deleterious effect on QoL in the physical domain, with lesser effects on social and mental functioning. This evidence of an early significant impact on their QoL reinforces recent recommendations for early treatment and primary prevention.
\end{abstract}

Q uality of life (QoL) is an important indicator of the burden of musculoskeletal (MSK) disease. ${ }^{1}$ The pattern and magnitude of the effect of MSK conditions on QoL are best assessed longitudinally in an inception cohort, ensuring that baseline data are collected before the condition has occurred. Because longitudinal studies in this area have included subjects with existing disorders (that is, patients in observational or interventional trials), little is known about the true impact of such illness on previously healthy subjects. ${ }^{2-4}$ Most investigations examining the potential drop in QoL induced by MSK disorders have compared patients with established disease with healthy controls in a cross sectional design. ${ }^{56}$ The difference between groups can provide some information about the existence and amount of any effect on QoL, but methodological problems limit the conclusions that can be drawn.

A group of subjects with no MSK problems during the follow up period is required to control for the natural evolution of QoL over time. Age and sex matching is necessary because most relevant QoL dimensions decrease with age in adults ${ }^{7}$ and are consistently lower in women than in men throughout adult life. Use of appropriate controls allows for assessment of time related variation in QoL, comparison between patients with MSK disorders and healthy subjects, and specific evaluation of QoL changes related to MSK disorders.

The goal of the present study is to test the hypothesis that people who develop an MSK disorder have a significant change in subsequent QoL compared with controls matched for age and sex. The nature of MSK illness, whether it is chronic or acute, and the influence of any comorbidities are taken into account. Unlike previous investigations, disorder-free baseline QoL data are available for all subjects.

\section{MATERIAL AND METHODS}

\section{Design of the SU.VI.MAX quality of life study}

Subjects were drawn from the study population of the SU.VI.MAX trial, a large, randomised, placebo controlled longitudinal investigation initiated in France in 1994 to quantify possible preventive effects of antioxidants, vitamins, and minerals in a general population of women aged 3560 years and men $45-60$ years. ${ }^{8}$ The characteristics of the participants are close to those of the national population, which allows generalisation of the results to the French adult population. SU.VI.MAX subjects of both sexes aged 4560 years at entry, who underwent clinical examination in 1996 and completed QoL questionnaires in 1996 and 1998, were eligible for the present quality of life study. Questionnaires were mailed to respondents biennially, and returned at the subsequent clinical examination.

Of 4882 subjects invited to take part in the quality of life study, 3759 were eligible for inclusion, while 1123 did not complete QoL questionnaire at both measurement times. Subjects who did not complete follow up of the quality of life study were still followed up on the SU.VI.MAX trial. They represented $23 \%$ of the initial sample and did not show differences in age, sex and comorbidity characteristics from those with QoL data available at follow up. Among eligible subjects 2557 presented with MSK at baseline, leaving a study sample of 1202 MSK disorder-free subjects forming an inception cohort. On the basis of clinical examinations, Minitel telematic network (a small terminal widely used in France as an adjunct to the telephone) and postal declarations over time, they were assigned to one of two groups at the end of follow up:

Abbreviations: ANOVA, analysis of variance; MSK, musculoskeletal; QoL, quality of life; SF-36, Short Form-36 
- Subjects who developed an MSK disorder during follow up (MSK disorder group)

- Subjects who remained free of an MSK disorder throughout (MSK disorder-free group).

A random selection process was used to age $(+/-1$ year) and sex match each subject in the MSK disorder group with two subjects in the MSK disorder-free group. The aim of matching was to control for the potentially confounding effects of age and sex on the relationship between onset of a MSK disorder and QoL.

\section{QoL assessment}

QoL was assessed using a validated French translation' of the Short Form-36 (SF-36) generic questionnaire, ${ }^{10}$ a widely used general health status measure. SF-36 consists of 36 items divided into eight dimensions of health. Each dimension is scored from 0 (worst) to 100 (best possible health status). ${ }^{7}$

SF-36 has been applied in general population surveys in many countries and age groups, and used for specific MSK diseases, including rheumatoid arthritis and osteoarthritis. ${ }^{5}$ SF-36 scores were used to derive utility values, by computing SF-6D scores according to a recently proposed algorithm. ${ }^{11}$

\section{Morbidity assessment}

Sociodemographic and morbidity data were obtained by questionnaire at baseline. Details of prior medical events, including rheumatic disorders (back pain, neck pain, thoracic pain), non-rheumatic disorders such as cardiovascular disease, cancer, diabetes, infectious disease requiring antibiotic administration, digestive disorder, asthma, and miscellaneous conditions were recorded. In addition, an open question allowed free wording to report health problems.

Medical follow up included (a) monthly self reports by mail or Minitel of any new diseases or symptoms (pain, swelling, stiffness, invalidity...), related medical consultations, admissions to hospital, and treatments; $(b)$ a clinical visit every second year at which physicians of subjects with major health events of any kinds were contacted to confirm the diagnosis. Investigations were made if the Minitel connection was broken for a long period of time, or if a participant failed to keep a follow up appointment.

Table 1 Baseline characteristics of subjects with a musculoskeletal (MSK) disorder and age and sex matched MSK disorder-free controls

\begin{tabular}{|c|c|c|c|}
\hline Characteristics & $\begin{array}{l}\text { MSK } \\
\text { disorder } \\
(n=310)\end{array}$ & $\begin{array}{l}\text { MSK disorder- } \\
\text { free } \\
(n=620)\end{array}$ & p Value \\
\hline Age (years), mean (SD) & $51.1(4.3)$ & $51.1(4.3)$ & $-\dagger$ \\
\hline Women & 37 & 37 & $-\dagger$ \\
\hline Living alone & 13 & 9 & 0.07 \\
\hline Professional status & & & 0.42 \\
\hline Professionals & 7 & 9 & \\
\hline Managerial & 59 & 54 & \\
\hline Workers & 15 & 15 & \\
\hline Unemployed/retired & 19 & 22 & \\
\hline Education level & & & 0.48 \\
\hline Primary & 35 & 38 & \\
\hline Secondary & 25 & 26 & \\
\hline Tertiary & 40 & 36 & \\
\hline \multicolumn{4}{|l|}{ Comorbidity } \\
\hline Cancer & 3 & 1 & 0.08 \\
\hline Infectious disease $\ddagger$ & 14 & 6 & 0.0001 \\
\hline Diabetes & 0.7 & 1 & 0.50 \\
\hline Cardiovascular & 5 & 3 & 0.14 \\
\hline Antioxidant supplementation & 49.4 & 51.1 & 0.26 \\
\hline
\end{tabular}

Results are percentages unless otherwise stated.

*Standard deviation; †matching variables; finfectious disease requiring antibiotic treatment.
Most answers collected were symptoms: tendonitis, disc herniation, back pain, neck pain. MSK diagnosis was exceptional.

Criteria for classification were built up according to location of disorders:

- Spinal disorder: back pain, neck pain, diffuse spine pain, disk herniation, vertebral osteoporosis

- Joint disorder: osteoarthritis (spine excluded), microcrystalline arthritis, arthritis (joint pain, rheumatism)

- Extra-articular disorder: capsulitis, tendinopathy, carpal tunnel syndrome, muscular pain.

The criteria were tested by a subsample analysis by two rheumatologists blinded to each other (CHR, FG), and further resolving discrepancy by consensus. One rheumatologist (CHR) completed the whole sample analysis. Difficult cases, ambiguous wording were solved jointly.

There was no bar to a single subject reporting several MSK disorders during follow up. Because conditions that fell into different categories were classified and counted as such, the number of disorders could exceed the number of subjects.

A distinction was made between acute and chronic disorders, based on the frequency of (and time interval between) reports. MSK disorders were considered acute when reported once or more over less than 3 months. Acute relapsing disorders were those reported twice, with more than 3 months between the first and second reports, and were taken into account as acute disorders. Conditions reported three or more times over more than 3 months were recorded as chronic.

\section{Analysis of data}

Subject characteristics were recorded using means and standard deviation (SD) or percentages. Groups were compared at baseline for sociodemographic characteristics, QoL scores, and comorbidity using the $\chi^{2}$ test for qualitative variables, Student's $t$ test, and analysis of variance (ANOVA) for quantitative variables. QoL scores were computed in every SF-36 dimension as recommended by the developer. ${ }^{10}$

The incidence of MSK disorders was calculated by personyears of exposure until the first report in each category. Acute (unique or relapse) and chronic disorders were reported separately.

The impact of onset of an MSK disorder on the way in which QoL changes over time was estimated by comparing the change in QoL score from baseline to follow up between the two groups. The analysis took account only of the first occurrence of a chronic MSK disorder (that is, the earliest in the follow up), and the last occurrence of an acute condition (that is, the most recent).

Baseline univariate analysis was used to identify variables that varied significantly between the two groups, and subsequent multivariate analysis using a linear mixed ANOVA model was then adjusted for those and matching variables. This approach allowed repeated measurement of the dependent variable- that is, QoL scores, and provided an estimate of the effect of independent variables, such as onset of an MSK condition, to be expressed as mean scores adjusted on covariates. The difference in QoL change between groups was estimated by testing the significance of the interaction term of MSK occurrence with group over time effect (repeated measurement). MSK disorders were tested both overall and by category (spine, joint, and extraarticular). In addition, an analysis was conducted within the group with MSK disorder to test for the effect on QoL of acute (unique or relapse) versus chronic conditions. If a subject reported both acute and chronic disorders, only the latter were considered. This ANOVA model was applied to the 
Table 2 Musculoskeletal (MSK) disorders reported

\begin{tabular}{|c|c|c|c|c|c|}
\hline & \multicolumn{3}{|l|}{ Total } & \multirow{2}{*}{$\begin{array}{l}\text { Acute } \\
\text { No }\end{array}$} & \multirow{2}{*}{$\begin{array}{l}\text { Chronic } \\
\text { No }\end{array}$} \\
\hline & No & Incidence* & $95 \% \mathrm{Cl}$ & & \\
\hline Spine disorders & 230 & 13.6 & 12.0 to 15.3 & 173 & 57 \\
\hline Neck pain & 46 & 3.1 & 2.3 to 4.0 & 30 & 16 \\
\hline Back pain & 135 & 8.5 & 7.2 to 9.9 & 106 & 29 \\
\hline Disk herniations & 51 & 3.4 & 2.5 to 4.4 & 37 & 14 \\
\hline Osteoporosis (stress fractures) & 5 & 0.3 & 0.1 to 0.6 & 4 & 1 \\
\hline Spine diffuse & 12 & 0.8 & 0.4 to 1.3 & 12 & 0 \\
\hline Joints disorders & 63 & 4.2 & 3.2 to 5.3 & 58 & 5 \\
\hline Osteoarthritis & 40 & 2.7 & 1.9 to 3.6 & 38 & 2 \\
\hline Microcrystalline arthritis & 7 & 0.5 & 0.2 to 0.9 & 6 & 1 \\
\hline Arthritis & 20 & 1.4 & 0.9 to 2.1 & 18 & 2 \\
\hline Extra-articular disorders & 70 & 4.6 & 3.6 to 5.7 & 44 & 26 \\
\hline Tendonitis & 67 & 4.4 & 3.4 to 5.5 & 44 & 23 \\
\hline Capsulitis & 2 & 0.1 & 0.0 to 0.3 & 0 & 2 \\
\hline Carpal tunnel syndrome & 2 & 0.1 & 0.0 to 0.3 & 0 & 2 \\
\hline
\end{tabular}

analysis of each QoL dimension score as a dependent variable.

Two levels of type 1 error threshold were used: $10 \%$ in univariate analysis to select candidate variables for adjustment in the multivariate analysis, and 5\% to determine statistical significance in the multivariate analysis.

Statistical analysis was performed using the SAS system (version 8.2). ${ }^{12}$

\section{RESULTS}

Of 3759 eligible subjects, a total of 2557 (68\%) respondents reported previous MSK disorders at inclusion, leaving 1202 MSK disorder-free subjects to participate in the longitudinal QoL study. Table 1 presents the sociodemographic characteristics of the subjects in this group who developed MSK and MSK disorder-free controls matched for age and sex. Their mean age was 51.1 years, and $37 \%$ were women. Differences appeared only for living alone $(p=0.07)$, cancer $(p=0.08)$, infectious disease $(p<0.0001)$. MSK occurrence did not differ according to antioxidant or placebo allocation group $(p=0.26)$. Average (SD) follow up was 28 (2.8) months, during which time 892 subjects remained healthy and 310 reported onset of one or more MSK conditions. The 310 subjects in the MSK disorder group were then randomly matched for age and sex with 620 out of 892 healthy controls for further analysis.

Reports of onset of an MSK disorder (table 2) in the follow up period reached 17.4 per 100 person-years of exposure. Most were spinal conditions, with an incidence of 13.6 per 100 person-years (predominantly back pain at 8.5 per 100 person-years) then extra articular disorders (4.6 per 100 person-years).

The first event in each category and subcategory was taken into account in calculating the incidence. If an event-for instance, back pain, occurred before another event-for instance, arthritis, only the first event- that is, back pain, was taken into account in the sum total of the MSK diseases.

Fewer subjects reported chronic rather than acute MSK disorders, whether unique or repeated (57 versus 173, respectively, in the spine pain category, 5 versus 58 in the joint category (osteoarthritis cases are mostly osteoarthritis flares), and 26 versus 44 in the extra-articular category).

Table 3 gives adjusted mean QoL scores in both groups, and the differences in change in scores over time. Baseline scores were similar in the two groups. At follow up in the MSK disorder group, only the bodily pain score had significantly decreased (a 10 point loss). The MSK disorder group exhibited a greater decrease than controls in scores for: physical functioning $(-1.2$ between-group difference in within-group change), bodily pain $(-7.4)$, vitality $(-2.6)$, and general health $(-1.8)$.

Within the MSK disorder group, changes in QoL over time differed between subjects who had an acute condition and those whose problem was chronic (table 4). The mean time between QoL assessment and the first report of a chronic condition was 17 months, and that for the last report of an acute condition was 15 months. Table 4 illustrates QoL scores adjusted for age, sex, living alone, infectious and cancer comorbidities at baseline and disclosed during follow up. The times between the report of an MSK disorder and QoL assessment, reductions in scores for physical functioning, role emotional, and social functioning dimensions were greater in the chronic subgroup, with a significant difference in QoL change between the subgroups $(-2.2,-8.4$, and -5.9 , respectively). Between-group differences in the change in bodily pain and vitality dimension scores did not reach significance $(-3.9$ versus acute, $\mathrm{p}=0.16$ and $-2.5, \mathrm{p}=0.13$, respectively). SF-6D utility scores did not differ either between groups or over time (tables 3 and 4 ).

\section{DISCUSSION}

This inception cohort study confirms previous cross sectional results $^{13}{ }^{14}$ that onset of an MSK disorder reduces QoL. The bodily pain dimension is most affected, with a 10 point loss on a $0-100$ scale over 28 months of follow up. According to Ware et al, a five point difference is sufficient to reflect clinically and socially relevant change. ${ }^{15}$ It confirms a cross sectional Dutch study results which showed a worse QoL in people with MSK diseases than in the general population, typically in physical dimensions of SF-36, with greater decrease with the coexistence of more than one MSK disease. ${ }^{16}$ SF-36 physical dimension scores were slightly lower than ours. This may well reflect the prevalent casesthat is, established diseases in which the disease impact is more severe than in incident cases-that is, with recent onset or occurrence.

To our knowledge, this study is the first longitudinal comparison between subjects with and without onset of MSK disorders, of QoL data collected from the same subjects before and after onset of an MSK disorder. Use of MSK disorder-free controls matched for age and sex strengthens the conclusions drawn.

Efforts were made to ensure that the incidence of MSK disorders in the study sample was representative of the general population. Indeed, many eligible subjects $(n=2257)$ had already had such a condition. Others have published 

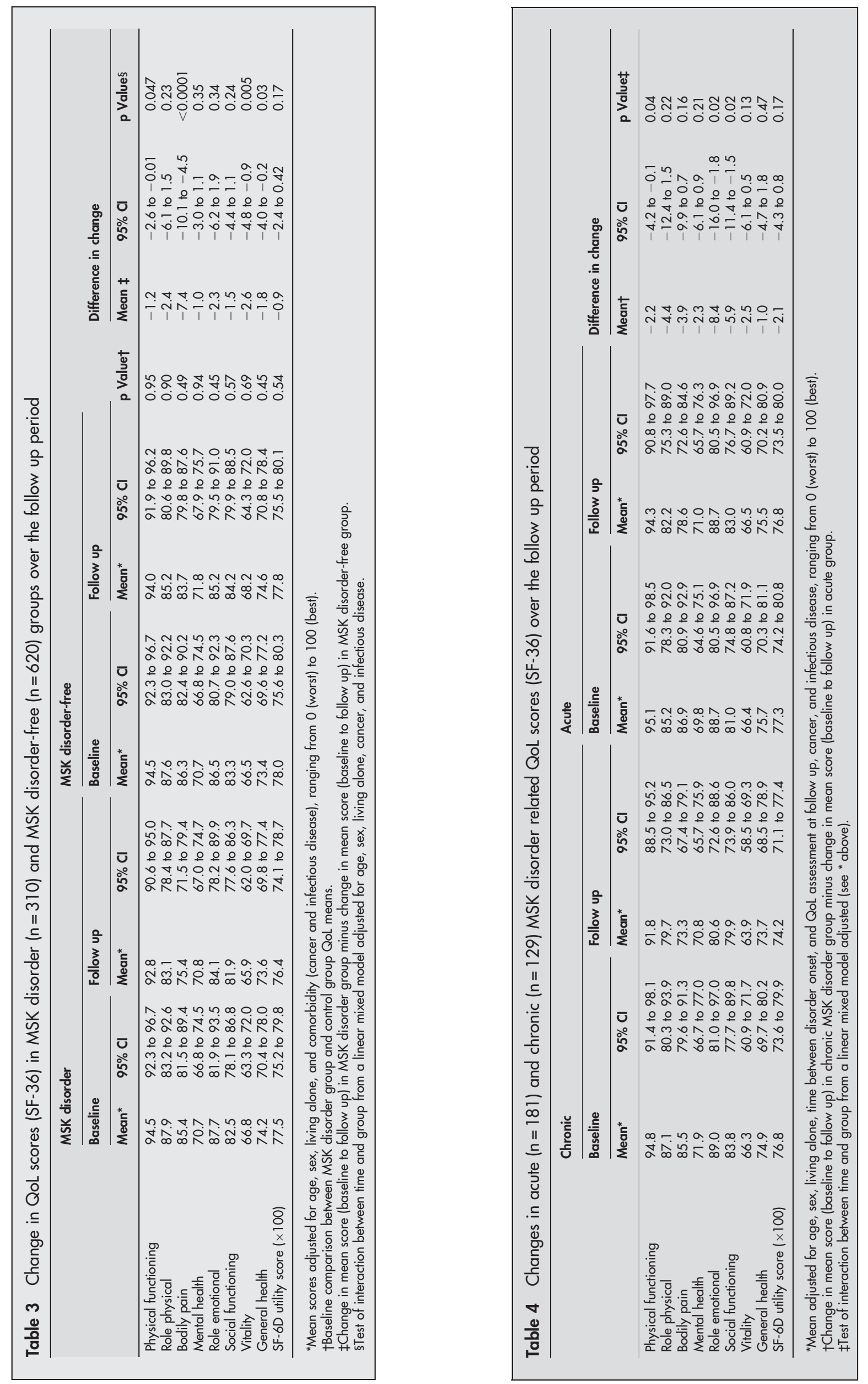
similar findings; for example, Hagen et al reported a prevalence of $60.8 \%$ among Norwegian adults in $1997 .{ }^{17}$

Moreover, the inclusion criteria applied here allowed for the identification of truly new cases, thus making it possible to calculate incidence rates and gain more information about within-subject changes in QoL over time.

In this 45-60 year age-group, the incidence of 17.4 per 100 person-years of exposure (time to occurrence) for any MSK disorder, and the annual incidence of back pain of 8.5 per 100 person-years, is within the range reported by previous European investigators. However, published estimates of the incidence of back pain vary widely. A Dutch study put it at $2-11 \%$ a year, depending on $a^{18}{ }^{18}$; a prospective study from Denmark reported a figure of $6 \%^{19}$; and a Swedish study estimated the monthly incidence to be $2.1 \%{ }^{20}$ Tendonitis and back pain are the most common, which is similar to the results from Picavet and Hazes of a higher incidence of tendonitis, capsulitis, and herniated discs between the ages of 45 and $64 .^{21}$

Although MSK conditions rarely cause death and are only seventh in the numbers of patients admitted to hospital, they are fifth for drug costs, third for chronicity, second for total health costs, first for health professional consultations, and are the most common disabling conditions in Western countries. $^{22}$ World wide, the proportion of the population disabled by rheumatism ranges from $2.8 \%$ in the United States to $8 \%$ in Great Britain. ${ }^{23-25}$ In Canada, MSK problems accounted for $1.7 \%$ of the 1986 gross national product, a higher figure than that for cancer. ${ }^{26}$

The longitudinal design of the present investigation allowed for the identification of subjects remaining free from MSK disorders over time, and for their QoL to be measured at baseline and follow up. Another strength of the protocol is that subjects and controls were matched for age and sex. This is important because age and sex are confounding factors. Most QoL dimension scores relevant here decrease with age among adults and are lower in women than men. Furthermore, MSK disorders tend to increase in prevalence with age, and are subject to sex differences. ${ }^{27}$ Mean baseline SF-36 scores were similar in the MSK disorder and control groups. Consequently, differences at follow up, after adjustment for comorbidity present at baseline or shown during follow up, can be expected to truly reflect the impact on QoL of MSK disorders, and allow its magnitude to be assessed.

The use of a generic instrument in the present survey is justified by the fact that it was carried out in an initially MSK disorder-free general population.

This study has several limitations. The reliability of self reporting of symptom and disease occurrence by questionnaire has some limitations that may affect the accuracy of recorded incidence of MSK disorders. Onset of symptoms is more likely reported than diagnosis, on the one hand, and some disorders already affecting patients at entry might have been omitted, on the other.

Consequently, the data gathered here cannot readily be compared with the findings of epidemiological studies using medical (ICD-10) taxonomy. Nevertheless, prevalence figures calculated from self reported information correlate with abnormalities observed by physicians. ${ }^{28}$ Moreover, because subjects may fail to self report a disorder repeatedly as it becomes chronic, there may be a tendency to underestimate the incidence of chronic disease and misclassify certain conditions. Another reason is that we consider healthy subjects and that reported manifestations are early symptoms of conditions (acute or chronic).

The mid-life age group of the study sample (45-60 years), in which $80 \%$ of subjects were still professionally active, might limit the generalisability of the present findings and the comparability with other population surveys on the occurrence of MSK. However, the levels of comorbidity in such populations are moderate and offer an opportunity to study the specific influence of MSK disorders on QoL with little interference from other diseases. MSK conditions, at least chronic ones, are relatively rare among subjects under 45, whereas people over 60 are more likely to have comorbidities with an influence on QoL. Older people are also more likely to live alone, have a low income, and be physically inactive.

Furthermore, as disorders were reported at regular intervals over the follow up period rather than as they occurred, acute conditions were more likely than chronic ones to be missed.

Although chronic disorders are well known to influence the psychosocial dimensions of the QoL, ${ }^{3}$ we found a low relative impact on mental and social functioning that may be attributable to the predominance of acute disorders. Comparison between acute and chronic MSK conditions bears this out: a significant between-group difference in change in SF-36 social functioning and role emotional dimensions was noted (the greater reduction was in chronic conditions), but the mental health dimension scores were close to those in normal subjects, as previously reported. ${ }^{13}$ The comparison of SF-6D utility scores between groups is limited by the use of English population weightings, which were the only ones available at this time.

Data on mean changes in QoL due to MSK disorders and baseline variance will help estimate the number of subjects required in future MSK prevention trials.

In conclusion, this inception investigation demonstrates that onset of an MSK disorder influences QoL. The physical domain is predominantly affected, but mental and social function are also impaired in comparison with control group findings. This evidence of an early significant impact on their QoL reinforces recent recommendations for early treatment and primary prevention.

\section{ACKNOWLEDGEMENT}

Grant support: Académie Nationale de Médecine (French National Academy of Medicine).

\section{Authors' affiliations}

C H Roux, F Guillemin, S Boini, F Longuetaud, S Briançon, EA 3444, Ecole de Santé Publique, Faculté de Médecine, Nancy, France

C H Roux, Service de Rhumatologie, CHU de Nice, France

N Arnault, S Hercberg, Unité 557-Unité Mixte de Recherche Inserm/

Inra/Cnam, 5 rue Vertbois, Paris, France

\section{REFERENCES}

1 Scott D, Garrood T. Quality of life measures: use and abuse. Baillieres Best Pract Res Clin Rheumatol 2000;14:663-87.

2 Bombardier C, Ware J, Russell I, Larson M, Chalmers A, Read J. Auranofin therapy and quality of life in patients with rheumatoid arthritis. Results of a multicentric trial. Am J Med 1986;81:565-78.

3 Ethgen O, Kahler K, Kong S, Reginster J, Wolfe F. The effect of health related quality of life on reported use of health care resources in patients with osteoarthritis and rheumatoid arthritis: a longitudinal analysis. J Rheumatol 2002;29:1147-55

4 Suurmeijer TP, Waltz M, Moum T, Guillemin F, Van Sonderen FL, Briançon S, et al. Quality of life profiles in the first years of rheumatoid arthritis: results from the EURIDISS longitudinal study. Arthritis Rheum 2001;45:111-21.

5 Hill C, Parsons J, Taylor A, Leach G. Health related quality of life in a population sample with arthritis. J Rheumatol 1999;26:2029-35.

6 Birrel F, Hassel A, Jones P, Dawes P. How does the Short Form 36 health questionnaire (SF36) in rheumatoid arthritis (RA) relate to RA outcome measures and SF-36 population values? A cross-sectional study. Clin Rheumatol 2000;19:195-9.

7 Ware J, Kosinski M, Keller S. SF36 physical \& mental health summary scales: a user's manual. Boston MA: The Health Institute, New England Medical Center, 1993.

8 Hercberg S, Preziosi P, Briançon S, Galan P, Triol I, Malvy D, et al. A primary prevention trial using nutritional doses of antioxidant vitamins and minerals in cardiovascular diseases and cancers in general population: the SU.VI.MAX study-design, methods, and participant characteristics. Control Clin Trials 1998;19:336-51. 
9 Leplège A, Ecosse E, Verdier A, Perneger T. The French SF-36 Health Survey: translation, cultural adaptation and preliminary psychometric evaluation. J Clin Epidemiol 1998;51:1013-23.

10 Ware J, Gandek B. Overview of the SF-36 Health Survey and the internationa quality of life assessment (IQOLA) project. J Clin Epidemiol 1998;51:903-12.

11 Brazier J, Roberts J, Deverill M. The estimation of a preference-based measure of health from the SF-36. J Health Econ 2002;21:271-92.

12 SAS Institute Inc. SAS/SAT user's guide, Release 8.2. Cary, NC: SAS Institute Inc, 1999.

13 Schlenk A, Erlen A, Dunbar-Jacob J, McDowell J, Engberg S, Sereika S, et al. Health-related quality of life in chronic disorders: a comparison across studies using the MOS SF-36. Qual Life Res 1998;7:57-65.

14 Sprangers A, de Regt E, Andries F, van Agt H, Bijl R, de Boer J, et al. Which chronic conditions are associated with better or poorer quality of life? J Clin Epidemiol 2000;53:895-907.

15 Ware JE, Snow K, Kosinski M, Gandek B. SF-36 Health Survey. In: Manual and interpretation guide. Boston MA: The Health Institute, New England Medical Center, 1993

16 Picavet HS, Hoeymans N. Health related quality of life in multiple musculoskeletal diseases: SF-36 and EQ-5D in the DMC3 study. Ann Rheum Dis 2004;63:723-9

17 Hagen K, Kvien T, Bjorndal A. Musculoskeletal pain and quality of life in patients with noninflammatory joint pain compared to rheumatoid arthritis: population survey. J Rheumatol 1997;24:1703-9.

18 Valkenburg HA, Haanen HCM. The epidemiology of low back pain. In: Withe AA III, Gordon S, eds. Symptoms on idiopathic low back pain. Miami, Florida: Mosby Company, 1982.
19 Biering-Soerensen F. Low back trouble in a general population of 30-, 40-, 50-, and 60-year old men and women. Dan Med Bull 1982;29:289-99.

20 Svensson $\mathbf{H}$, Andersson $\mathrm{G}$. Low back pain in forty-seven year old men. Frequency of occurence and impact on medical services. Scand J Rehab Med 1982; 14:47-53.

21 Picavet H. S, Hazes JMW. Prevalence of self reported musculoskeletal diseases is high. Ann Rheum Dis 2003:62:644-50.

22 Glazier R, Fry J, Badley E. Arthritis and rheumatism are neglected health priorities: a bibliometric study. J Rheumatol 2001;28:706-11.

23 Centers for Disease Control and Prevention. Arthritis prevalence and activity limitations. United States, 1990. MMWR 1994:43:433-8.

24 Reynolds D, Chambers L, Badley E, Bennett K, Goldsmith C, Jamieson E, et al. Physical disability among Canadians reporting musculoskeletal diseases. J Rheumatol 1992;19:1020-30.

25 Badley E, Tennant A. Impact of disablement due to rheumatic disorders in a British population: estimates of severity and prevalence from the Calderdale rheumatic disablement survey. Ann Rheum Dis 1993;52:6-13.

26 Badley $\mathbf{E}$. The economic burden of musculoskeletal disorders in Canada is similar to that for cancer and may be higher. $J$ Rheumatol 1995;22:204-6.

27 Symmons D. Population studies of musculoskeletal morbidity. In: Alan J Silman, Marc C Hochberg, eds. Epidemiology of the rheumatic diseases. Oxford: Oxford University Press, 2001:1-28.

28 Cunningham L, Kelsey J. Epidemiology of musculoskeletal impairments and associated disability. Am J Public Health 1984;74:574-9. 